medgen $2009 \cdot 21: 231-236$

DOI 10.1007/s11825-009-0160-0

Online publiziert: 15. Juli 2009

(c) Springer Medizin Verlag 2009

A. Tzschach

Max-Planck-Institut für molekulare Genetik, Berlin

\title{
Genetik der nichtsyndromalen geistigen Behinderung
}

onen in einem Gen von einem charakteristischen syndromalen Phänotyp bis hin zu unspezifischer MR reichen (z. B. RSK2Mutationen bei Coffin-Lowry-Syndrom und nichtsyndromaler X-chromosomaler mentaler Retardierung).

In den letzten Jahren wurden zahlreiche neue NS-MR-Gene auf dem XChromosom entdeckt, und auch bei den autosomalen Formen der NS-MR sind erste Fortschritte zu verzeichnen. Dieser Artikel soll - mit Schwerpunkt auf der diagnostischen Relevanz einzelner Gene und Untersuchungsmethoden - einen Überblick über den aktuellen Wissensstand und einen Ausblick auf die für die nähere Zukunft absehbaren Entwicklungen zu diesem Themengebiet geben.

\section{X-chromosomale mentale Retardierung (XLMR)}

Knaben sind signifikant häufiger von geistiger Behinderung betroffen als Mädchen (Geschlechterverhältnis männlich:weiblich=1,4:1 für schwere MR und 1,9:1 für milde MR). Diese Diskrepanz erklärt sich z. T. durch Gendefekte auf dem X-Chromosom. Die Daten aus aktuellen Studien legen nahe, dass bei etwa 10\% aller männlichen MR-Patienten ein X-chromosomaler Gendefekt die Ursache ist [4].

Derzeit sind mehr als 80 Gene für Xchromosomale mentale Retardierung bekannt [3, 13] [15], von denen viele die Ursache syndromaler Erkrankungen [z. B. ATRX( $\alpha$-Thalassämie mentale Retardierung)-Syndrom, Menkes-Syndrom] sind. Die Aufklärung von Gendefekten bei nichtsyndromaler XLMR wurde durch
Kopplungsuntersuchungen in Familien mit X-chromosomalem Erbgang und die koordinierte Erfassung solcher Familien in internationalen Forschungskonsortien erleichtert. Alle XLMR-Gene sind jedoch - mit Ausnahme von FMR1 - nur bei einem sehr geringen Prozentsatz der Patienten verändert. Diese genetische $\mathrm{He}$ terogenität macht insbesondere bei sporadischem Auftreten von MR ein sinnvolles Mutationsscreening in der Routinediagnostik derzeit nahezu unmöglich. Patienten mit Hinweisen auf familiäre XLMR, d. h. wenn es mindestens einen weiteren betroffenen Bruder oder andere betroffene männliche Verwandte in der mütterlichen Linie gibt, sollte die $\mathrm{Mu}$ tationsanalyse im Rahmen laufender Forschungsprojekte angeboten werden (z. B. EUROMRX-Konsortium, Infobox 1).

Bei einigen XLMR-Genen sind $\mathrm{Mu}-$ tationen ausschließlich mit nichtsyndromaler MR assoziiert (ILRAPL1, TM4SF2, ZNF674, ZNF41, ZNF81, FTSJ1, DLG3, FMR2, RPL10, GDI1, ACSL4, PAK 3 , AGTR2, ARHGEF6). Mutationen in mehreren anderen Genen (z. B. FMR1, $A R X$, $P Q B P_{1}, M E C P_{2}, C U L_{4} B, M C T 8, A P_{1} S_{2}$, $\left.S L C 6 A 8, O P H N_{1}, S L C_{9} A 6\right)$ gehen hingegen häufig mit zusätzlichen klinischen Merkmalen (z. B. Epilepsie, Mikrozephalie, Spastik) einher. Auch wenn sie meist keine „Syndrome“ im klassischen Sinne verursachen, sollten die im Folgenden besprochenen Gene und Genveränderungen bei MR-Patienten mit entsprechenden Begleitsymptomen differenzialdiagnostisch in Betracht gezogen werden. 


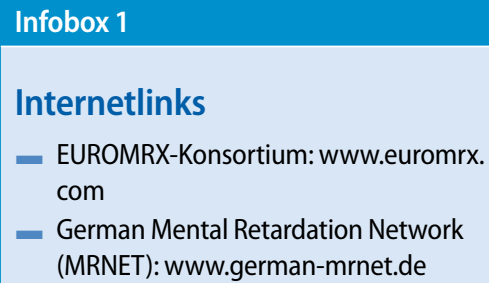

\section{FMR1 (Fragiles-X-Syndrom)}

Das Fra-X-Syndrom ist die mit Abstand häufigste XLMR-Form (bis zu 25\% aller XLMR-Familien und etwa $2 \%$ bei sporadischen männlichen MR-Patienten) und wird ausführlich in dem Weiterbildungsartikel von P. Steinbach in diesem Heft besprochen. Hier soll der Hinweis genügen, dass die über die Entwicklungsverzögerung hinausgehenden klinischen Zeichen vor der Pubertät meist wenig ausgeprägt sind, es sich also in diesem Alter eher um eine nichtsyndromale MR-Form handelt, und dass das Fragile-X-Syndrom differenzialdiagnostisch auch bei älteren Patienten mit unspezifischem Phänotyp mit in Erwägung gezogen werden muss.

\section{ARX (Hirnfehlbildungen mit Epilepsie und Genital- fehlbildungen, XLAG)}

Mutationen im $A R X-G e n$ sind mit einer Häufigkeit von etwa $5 \%$ in XLMRFamilien nach dem Fra-X-Syndrom die zweithäufigste Ursache für X-chromosomale geistige Behinderung. Neben Patienten mit nichtsyndromaler MR wurden $A R X$-Mutationen auch bei Patienten mit West-Syndrom (Epilepsie, infantile Spasmen), Partington-Syndrom (Epilepsie, dystone Handbewegungen, Dysarthrie), Proud-Syndrom (intersexuelles Genitale und Corpus-callosum-Agenesie) und dem XLAG-Syndrom („X-linked lissencephaly and ambiguous genitalia“) nachgewiesen (s. auch den Beitrag von G. Uyanik und U. Hehr in diesem Heft). Patienten mit MR und neurologischen Symptomen haben häufig eine rekurrente, aber das Leseraster beibehaltende 24-bp-Duplikation (c.428-451) im ARX-Gen; bei Patienten mit XLAG sind Funktionsverlustmutationen die Ursache.

Abgesehen von den eindeutig syndromalen Formen mit Hirn- und Genitalfehl- bildungen sollte in der Routinediagnostik ein $A R X$-Mutationsscreening auch bei sporadischen MR-Patienten mit Epilepsie oder infantilen Spasmen sowie bei XLMR-Familien in Betracht gezogen werden. Bei sporadischen nichtsyndromalen männlichen MR-Patienten ist die Prävalenz von $A R X$-Mutationen jedoch sehr gering und eine routinemäßige Analyse derzeit nicht gerechtfertigt.

\section{PQBP1 (Mikrozephalie, Renpenning-Syndrom)}

Patienten mit Mutationen in $P Q B P_{1}$ sind klinisch durch Mikrozephalie, Kleinwuchs, Spastik und seltener auch durch Herzfehler, Augenfehlbildungen (Mikrophthalmie), kleines Hodenvolumen, Gaumenspalte sowie Analatresie gekennzeichnet, wobei eine erhebliche inter- und intrafamiliäre Variabilität besteht [8]. PQBP1-Mutationen sind die Ursache mehrerer sich klinisch überlappender Syndrome, die ursprünglich anhand einzelner großer Familien phänotypisch definiert wurden [Renpenning-Syndrom, Sutherland-Haan-Syndrom, Golabi-Ito-Hall-Syndrom, zerebropalatokardiales (Hamel) Syndrom]. Bei mikrozephalen männlichen Patienten, die zusätzlich eines der oben angeführten Merkmale aufweisen, ist ein PQBP1-Mutationsscreening auch in der Routinediagnostik gerechtfertigt.

\section{MECP2-Duplikationen (MR und Enzephalopathie)}

Mutationen im $\mathrm{MECP}_{2}$-Gen wurden zunächst als Ursache des Rett-Syndroms, eines auf das weibliche Geschlecht beschränkten schweren MR-Syndroms mit charakteristischem Krankheitsverlauf und neurologischen Symptomen, beschrieben.

MECP2-Punktmutationen wurden in seltenen Fällen auch bei männlichen $\mathrm{Pa}$ tienten mit schwerer Enzephalopathie nachgewiesen [17].

Eine wesentlich größere Rolle spielen aber Duplikationen des $\mathrm{MECP}_{2}$-Gens bei Knaben mit schwerer mentaler Retardierung. Klinisch sind diese Patienten durch schwere MR, infantile Hypotonie, progressive Spastik, Ataxie, Epilepsie, feh- lende Sprachentwicklung und Immundefekte charakterisiert.

$M E C P_{2}$-Duplikationen sind eine der häufigeren Ursachen für XLMR; sie sind für 1\% der Familien mit X-chromosomaler geistiger Behinderung verantwortlich und haben bei sporadischen Patienten mit schwerer Enzephalopathie sogar eine Häufigkeit von 2\% [9]. Bei Mädchen bzw. Frauen scheinen sie keine Krankheitssymptome zu verursachen.

Für die Praxis bedeutet dies, dass bei Knaben mit schwerer MR, Enzephalopathie, Hypotonie und/oder Spastik eine $M E C P_{2}$-Duplikation ausgeschlossen werden sollte (eine Mutationsanalyse in $M E$ $\mathrm{CP}_{2}$ ist nicht ausreichend!). Diese Duplikationen sind auch mit hochauflösender Array-CGH nachweisbar. Aufgrund der sehr schweren MR und der Ataxie sollten $M E C P 2$-Duplikationen auch als Differenzialdiagnose zum Angelman-Syndrom in Erwägung gezogen werden.

\section{CUL4B (Kleinwuchs, relativer Makrozephalus, Adipositas)}

Patienten mit $C U L_{4} B$-Mutationen sind durch Kleinwuchs, relativen Makrozephalus, Adipositas, Anfallsleiden, Hypogonadismus und Tremor gekennzeichnet; der Grad der geistigen Behinderung kann erheblich variieren. Klinisch bestehen Überlappungen zum Prader-Williund zum Börjeson-Forssman-LehmanSyndrom. Mit bereits jetzt 9 publizierten Mutationen scheint $C U L_{4} B$ zu den häufiger betroffenen XLMR-Genen zu gehören [14].

\section{MCT8/SLC16A2}

(erhöhtes Serum-fT3, Allan-Herndon-Dudley-Syndrom)

MCT8 (SLC16A2) kodiert für einen neuronalen Schilddrüsenhormontransporter. Laborchemisch führen Mutationen in diesem Gen zu Verschiebungen der Schilddrüsenparameter im Blut: erhöhtes freies Trijodthyronin ( $\mathrm{fT}_{3}$ ), niedrignormales bis erniedrigtes freies Thyroxin ( $\mathrm{fT}_{4}$ ), aber normales TSH (Thyreoidea stimulierendes Hormon).

Klinisch verursachen MCT8-Mutationen einen als Allan-Herndon-DudleySyndrom bezeichneten neurologischen 
Symptomenkomplex aus schwerer MR, Hypotonie, verminderter Muskelmasse, Spastik und choreatischen Bewegungen. Bei männlichen Patienten mit schwerer MR und den oben genannten neurologischen Beschwerden sollten eine $\mathrm{fT}_{3}-\mathrm{Be}-$ stimmung veranlasst und bei erhöhten $\mathrm{fT}_{3}$-Werten ein MCT8-Mutationsscreening durchgeführt werden [5].

\section{AP1S2 (Basalganglienverkalkung)}

Patienten mit AP1S1-Mutationen haben außer einer sehr variablen geistigen Behinderung eine Basalganglienverkalkung, erhöhte Proteinwerte im Liquor und teilweise auch Hydrozephalus, Spastik, Hypotonie, aggressives Verhalten oder $\mathrm{Au}$ tismus [1]. Wenngleich bis jetzt nur 7 $A P_{1} S_{2}$-Mutationen beschrieben wurden, sollte insbesondere bei Patienten mit ungeklärter Proteinerhöhung im Liquor sowie bei Patienten mit Basalganglienverkalkung als Differenzialdiagnose zu disruptiven Hirnfehlbildungen infolge intrauteriner Infektionen und zu Aicardi-Goutières-Syndrom eine Mutationsanalyse in diesem Gen erwogen werden.

\section{SLC6A8 (Kreatintransporterstörung)}

SLC6A8-Mutationen führen zu schwerer MR, Kleinwuchs, Hypotonie, verminderter Muskelmasse und häufig auch Epilepsie. SLC6A8 kodiert für einen neuronalen Kreatintransporter, dessen Funktionsverlust einerseits zu einer verminderten Kreatinaufnahme in Nervenzellen führt (was mittels Protonenmagnetresonanzspektroskopie - 1 H-MRS - messbar ist), andererseits auch ein laborchemisch nachweisbar erhöhtes Verhältnis von Kreatin zu Kreatinin im Urin zur Folge hat. Mutationen in SLC6A8 zählen zu den häufigeren Ursachen von XLMR und sollten insbesondere bei auffälligen Kreatin-Kreatinin-Werten und der komplexen Symptomatik als Differenzialdiagnose berücksichtigt werden.

\section{OPHN1 (Kleinhirnhypoplasie)}

Das charakteristische Merkmal von Patienten mit $O P H_{1}$-Mutationen ist die Hypoplasie des Kleinhirns (insbesondere der Vermis), darüber hinaus finden sich

medgen $2009 \cdot 21: 231-236 \quad$ DOI 10.1007/s11825-009-0160-0

(c) Springer Medizin Verlag 2009

\section{A. Tzschach \\ Genetik der nichtsyndromalen geistigen Behinderung}

\section{Zusammenfassung}

Die meisten Patienten mit mentaler Retardierung (MR) sind von nichtsyndromaler MR (NS-MR) betroffen, $d$. h. sie weisen entweder überhaupt keine zusätzlichen klinischen, radiologischen oder metabolischen Merkmale auf oder die weiteren Auffälligkeiten sind für die Zuordnung zu einem etablierten Fehlbildungssyndrom nicht spezifisch genug. In den letzten Jahren wurden erhebliche Fortschritte bei der Aufklärung X-chromosomal vererbter Formen der NS-MR erzielt, und auch bei der Erforschung autosomaler NS-MR sind erste Erfolge zu verzeichnen. Beide Formen sind durch ausgeprägte genetische Heterogenität gekennzeichnet. Eine routinemäßige Mutationsanalyse in den bekannten NS-MR-Genen ist derzeit bei sporadischen Patienten nur be- grenzt möglich und wird erst mit der Einführung neuer Sequenziertechnologien breite Anwendung finden. Im Gegensatz dazu können Patienten mit familiärer NS-MR bereits jetzt in molekulargenetische Screeningprogramme eingeschlossen werden. In der aktuellen Routinediagnostik sind aufgrund klinischer Überlappungen mit syndromalen MR-Formen auch mehrere Gene für die Xchromosomale NS-MR von Bedeutung.

\section{Schlüsselwörter}

Nichtsyndromale mentale Retardierung . X-chromosomale MR - Autosomal-rezessive MR $\cdot$ Autosomal-dominante MR $\cdot$ Wiederholungsrisiko

\section{Genetics of nonsyndromic mental retardation}

\section{Abstract}

Most patients with mental retardation (MR) are nonsyndromic; i.e. they either have no accompanying clinical, radiological, or metabolic abnormalities, or their additional features are not specific enough to enable allocation to a recognizable malformation syndrome. Numerous novel disease genes for X-chromosomal nonsyndromic MR (NS-MR) have been elucidated in recent years, and research into autosomal forms of NS-MR has yielded first results. Both forms have turned out to be characterized by extreme genetic heterogeneity. Routine diagnostic mutation screening in the known NS-MR genes is currently not feasible in sporadic patients but will be facilitated by novel sequencing technologies in the near future. Patients with familial NS-MR should be offered inclusion in ongoing research programmes. Several X-chromosomal NS-MR genes demand consideration in the routine diagnostic workup of MR patients because they overlap phenotypically with syndromic forms of MR.

\section{Keywords}

Nonsyndromic mental retardation $\cdot X$-linked MR - Autosomal-recessive MR - Autosomaldominant MR $\cdot$ Recurrence risk 
auch vergrößerte Hirnventrikel, Epilepsie und Hypogenitalismus. Trotz der offenbar geringen Häufigkeit von $O P H_{1}$ Mutationen sollte ein Mutationsscreening in diesem Gen bei männlichen MR-Patienten mit Kleinhirnhypoplasie erwogen werden.

\section{SLC9A6 (Angelman-Syndrom- ähnliche XLMR)}

SLC9A6-Mutationen verursachen ein XLMR-Krankheitsbild, das phänotypische Überlappungen mit dem Angelman-Syndrom besitzt: Neben einer schweren MR leiden die Betroffenen an Mikrozephalie, Ataxie, Epilepsie und fehlender Sprachentwicklung. Bis jetzt wurden in diesem erst kürzlich entdeckten Gen $4 \mathrm{Mu}$ tationen nachgewiesen, angesichts des relativ spezifischen klinischen Bildes könnte dieses Gen in die routinemäßigen molekulargenetischen Untersuchungen bei Angelman-Syndrom aufgenommen werden.

\section{Autosomal-rezessive mentale Retardierung}

Die Aufklärung von Gendefekten bei nichtsyndromaler autosomal-rezessiver geistiger Behinderung (NS-ARMR) begann verhältnismäßig spät: Der erste Genlocus für autosomal-rezessive NS-MR wurde im Jahre 2000 in einer konsanguinen deutschstämmigen Familie aus einem religiösen Isolat in den USA nachgewiesen, und das erste NS-ARMR-Gen PRSS12 - wurde 2002 publiziert.

Dieses scheinbar späte Interesse an NSARMR mag angesichts der hohen Prävalenz von NS-MR überraschen, ist aber wohl in dem unspezifischen Phänotyp, der (wie inzwischen bekannt ist) extremen Heterogenität und den in westlichen Ländern kleinen Familiengrößen sowie der geringen Häufigkeit von Verwandtenehen begründet. Hinweise auf die Bedeutung autosomal-rezessiver Gendefekte für NS-MR gab es gleichwohl aufgrund der deutlich erhöhten Prävalenz von MR in Ländern mit hohem Anteil konsanguiner Ehen bereits viel früher. Es ist aber auch jetzt noch weitgehend unklar, wie hoch der Anteil autosomal-rezessiver Ursachen an dem Gesamtkollektiv mental retardierter Patienten in unserer Bevölkerung ist.

Blutsverwandtschaft der Eltern und eine große Zahl betroffener und gesunder Geschwister erleichtern die Kartierung von autosomal-rezessiven Genorten, und aus diesem Grund sind auch alle bis jetzt publizierten 5 ARMR-Gene und 7 Genloci in großen konsanguinen Familien aufgeklärt worden. Im Gegensatz zu anderen Erkrankungen, in denen Mutationen in einzelnen Hauptgenen für einen größeren Prozentsatz der Patienten ursächlich sind (z. B. GJB2 bei autosomalrezessiver Taubheit oder ASPM bei autosomal-rezessiver Mikrozephalie) und die damit auch sinnvoll in der Routinediagnostik untersucht werden können, ist NS-ARMR durch ausgeprägte genetische Heterogenität gekennzeichnet [11]. Lediglich in einem Gen - TUSC 3 - wurden bislang 2 verschiedene Mutationen in unabhängigen Familien publiziert, und das seltene Auftreten überlappender Kopplungsintervalle, die im Rahmen einer Studie an mehr als 200 konsanguinen Familien aus dem Iran identifiziert wurden, legt nahe, dass kein Gen für mehr als $2 \%$ der NSARMR-Patienten verantwortlich ist (Kuss et al., persönliche Mitteilung).

Auch die Funktionen der Produkte der bis jetzt bekannten Gene sind vielfältig und spiegeln die Komplexität des Gehirns wider: PRSS1 (Neurotrypsin) ist eine trypsinähnliche Serin-Protease, CRBN (Cereblon) ist ein Modulator für einen Kaliumkanal, CC2D1A ist ein Endozytoseregulator und spielt eine Rolle im endosomalen Trafficking, GRIK2 ist ein Glutamatrezeptor, und TUSC 3 hat möglicherweise bei der N-Glykosilierung von Proteinen eine Funktion.

In der Routinediagnostik sporadischer Patienten mit Verdacht auf NS-ARMR (der sich in der Regel aus einer Konsanguinität der Eltern ergibt) spielen die angeführten Gene noch keine Rolle. Ein sinnvolles Mutationsscreening für diese und die zu erwartenden zahlreichen weiteren NS-ARMR-Gene wird erst mit preiswerteren Technologien möglich sein, welche die parallele Untersuchung mehrerer Gene erlauben. Familien mit 2 oder mehr Betroffenen kann aber bereits jetzt der Einschluss in laufende Forschungsprojekte angeboten werden (z. B. am MPI für molekulare Genetik Berlin, Abteilung Ropers).

\section{Autosomal-dominante mentale Retardierung}

Autosomal-dominante Neumutationen sind wahrscheinlich für einen erheblichen Prozentsatz insbesondere der sporadisch auftretenden MR-Fälle verantwortlich. Da sich aber Patienten mit schweren MR-Formen nur in Ausnahmefällen fortpflanzen, stehen praktisch keine ausreichend großen Stammbäume für Kopplungsanalysen zur Verfügung. Unsere Kenntnisse über einzelne dominante MR-Gene beruhen daher fast ausschließlich auf der Untersuchung chromosomaler Aberrationen. Eine wichtige Informationsquelle stellte und stellt dabei die Bruchpunktanalyse bei Patienten mit neu entstandenen balancierten Rearrangements (z. B. Translokationen) dar. Die Identifizierung unterbrochener Gene an den chromosomalen Bruchpunkten führte nicht nur zur Aufklärung zahlreicher syndromaler Krankheitsbilder, sondern definierte auch Kandidatengene für nichtsyndromale $\mathrm{MR}$, insbesondere wenn ein Gen bei mehreren nichtverwandten Patienten betroffen war (z. B. AUTS2) [9].

Die Einführung der Array-CGH, die erstmals den ungezielten Nachweis auch sehr kleiner chromosomaler Deletionen oder Duplikationen ermöglichte, leitete große Fortschritte bei der Aufklärung autosomal-dominanter Ursachen ein (s. auch den Beitrag von A. Reis und A. Rauch in diesem Heft). Die meisten submikroskopischen chromosomalen Imbalancen umfassen zwar mehrere Gene, aber bei sehr kleinen Veränderungen ist teilweise nur ein einziges Gen direkt betroffen. Durch die systematische Erfassung solcher Aberrationen im Rahmen von Forschungsverbünden [z. B. MRNET („German mental retardation network“)] und zunehmend auch durch den Nachweis in der Routinediagnostik wird in den nächsten Jahren die Zahl der (Kandidaten)-Gene für autosomal-dominante NS-MR deutlich zunehmen.

Eine weitere Strategie zur Aufklärung autosomal-dominanter Gendefekte ist die Analyse funktioneller Kandidatengene. Im Prinzip kann zwar jedes neuro- 
nal exprimierte Gen als MR-Kandidatengen betrachtet werden, besonderes Interesse verdienen aber Gene, die für Proteine in den Synapsen kodieren. $\mathrm{Zu}$ diesen zählt SYNGAP1, das für ein RasGTPase aktivierendes Synapsenprotein kodiert, und in SYNGAP1 wurden vor kurzem Mutationen bei Patienten mit schwerer MR und Epilepsie nachgewiesen [6]. Da aber die Suche nach Punktmutationen in großen Patientenkohorten mit den derzeitigen Methoden sehr aufwändig ist, dürfen wohl erst mit der Einführung leistungsstärkerer Sequenziertechnologien größere Fortschritte auf diesem Gebiet zu erwarten sein.

\section{Polygene und multifaktorielle Ursachen}

Mildere Formen der geistigen Behinderung [Intelligenzquotient (IQ) 50-70] stellen teilweise den unteren Bereich der Normalverteilung der Intelligenz dar. Während man bei Patienten mit schwerer MR (Prävalenz etwa 0,3-0,5\%) davon ausge- hen kann, dass die Hirnfunktionsstörung zu einem großen Teil Folge des Funktionsverlustes eines oder mehrerer Gene ist, wird als Ursache für die wesentlich häufigeren milden MR-Formen neben monogenen und chromosomalen Defekten auch ein Zusammenspiel von ungünstigen Genvarianten (Polymorphismen) und Umwelteinflüssen vermutet. $\mathrm{Zu}$ den daran beteiligten hypothetischen „Intelligenzgenen“" ist aber praktisch nichts bekannt. Assoziationsstudien wurden und werden durch die starke nichtgenetische Beeinflussung des Merkmals "Intelligenz" erschwert und erbrachten bislang keine befriedigenden Ergebnisse [2].

\section{Wiederholungsrisiko für Geschwister}

Für Geschwister von MR-Patienten mit nachgewiesener Genveränderung oder Chromosomenstörung lässt sich das Wiederholungsrisiko meist präzise angeben, und den Eltern kann in der Regel auch ein vorgeburtlicher Test angeboten werden.
Bei sporadischen NS-MR-Patienten ohne nachgewiesene Ursache muss auf empirische Zahlen zurückgegriffen werden, und das Wiederholungsrisiko beträgt für jedes Geschwister - mit geringen Schwankungen abhängig vom Geschlecht - etwa 8\% [16]. Für die Eltern ist diese Angabe allerdings meist unbefriedigend, da das tatsächliche Wiederholungsrisiko zwischen $50 \%$ und dem allgemeinen Basisrisiko für MR von etwa $2 \%$ liegen kann. In Unkenntnis des ursächlichen Gendefektes bei dem Indexpatienten kann auch keine zielgerichtete Pränataldiagnostik angeboten werden.

\section{Ausblick}

Nichtsyndromale geistige Behinderung ist ein Krankheitsbild mit ausgesprochen heterogenen genetischen Ursachen, was in der Komplexität des zentralen Nervensystems und der Tatsache, dass mehr als 50\% aller Gene im Gehirn exprimiert werden, begründet liegt. Trotz der hohen Prävalenz von NS-MR sind bis jetzt nur verhältnismäßig wenige Krankheitsgene identifiziert worden. Neue Tech-

\section{Hier steht eine Anzeige.}


mangenetikers wird es dann sein, anhand geeigneter Kriterien (klinisches Bild, Genfunktion, Vergleich mit ähnlichen Mutationen im selben Gen usw.) die einzelnen Mutationen auf ihre pathogenetische Relevanz hin zu beurteilen und daraus die richtigen Schlussfolgerungen für die genetische Beratung und ggf. auch die Pränataldiagnostik abzuleiten. Eine vergleichbare Situation liegt bereits jetzt in der Diagnostik mittels hochauflösender Array$\mathrm{CGH}$ vor, bei der sich nachgewiesene Aberrationen nicht immer eindeutig interpretieren lassen.

Solange jedoch derartige globale molekulargenetische Analysen für die Routinediagnostik nicht zur Verfügung stehen, bleibt es weiterhin die vordringliche Aufgabe des klinischen Genetikers und Syndromologen, bei idealerweise allen Patienten mit einem klinisch erkennbaren Krankheitsbild - insbesondere auch bei denen mit subtiler Ausprägung - die richtige Syndromdiagnose zu stellen und damit den Anteil der nicht klassifizierbaren, also nichtsyndromalen Patienten mit mentaler Retardierung möglichst gering zu halten.

\section{Korrespondenzadresse}

\section{Dr. A. Tzschach}

Max-Planck-Institut für molekulare Genetik Ihnestraße 73, 14195 Berlin

tzschach@molgen.mpg.de

Interessenkonflikt. Der korrespondierende Autor gibt an, dass kein Interessenkonflikt besteht.

\section{Literatur}

1. Borck G, Molla-Herman A, Boddaert N et al (2008) Clinical, cellular and neuropathological consequences of AP1S2 mutations: further delineation of a recognizable X-linked mental retardation syndrome. Hum Mutat 29(7):966-974

2. Butcher LM, Davis OS, Craig IW et al (2008) Genome-wide quantitative trait locus association scan of general cognitive ability using pooled DNA and $500 \mathrm{~K}$ single nucleotide polymorphism microarrays. Genes Brain Behav 7(4):435-446

3. Chiurazzi P, Schwartz CE, Gecz J et al (2008) XLMR genes: update 2007. Eur J Hum Genet 16(4):422434

4. De Brouwer AP, Yntema HG, Kleefstra T et al (2007) Mutation frequencies of $X$-linked mental retardation genes in families from the EuroMRX Consortium. Hum Mutat 28(2):207-208

5. Frints SG, Lenzner S, Bauters M et al (2008) MCT8 mutation analysis and identification of the first female with Allan-Herndon-Dudley syndrome due to loss of MCT8 expression. Eur J Hum Genet 16(9):1029-1037

6. Hamdan FF, Gauthier J, Spiegelman D et al (2009) Mutations in SYNGAP1 in autosomal nonsyndromic mental retardation. N Engl J Med 360(6):599605

7. Hunter AG (2000) Outcome of the routine assessment of patients with mental retardation in a genetics clinic. Am J Med Genet A 90(1):60-68

8. Kalscheuer VM, Freude K, Musante L et al (2003) Mutations in the polyglutamine binding protein 1 gene cause X-linked mental retardation. Nat Genet 35(4):313-315

9. Kalscheuer VM, FitzPatrick D, Tommerup $\mathrm{N}$ et al (2007) Mutations in autism susceptibility candidate 2 (AUTS2) in patients with mental retardation. Hum Genet 121(3-4):501-509

10. Lugtenberg D, Kleefstra T, Oudakker AR et al (2008) Structural variation in Xq28: MECP2 duplications in $1 \%$ of patients with unexplained XLMR and in $2 \%$ of male patients with severe encephalopathy. Eur J Hum Genet

11. Najmabadi H, Motazacker MM, Garshasbi M et al (2007) Homozygosity mapping in consanguineous families reveals extreme heterogeneity of non-syndromic autosomal recessive mental retardation and identifies 8 novel gene loci. Hum Genet 121(1):43-48

12. Rauch A, Hoyer J, Guth S et al (2006) Diagnostic yield of various genetic approaches in patients with unexplained developmental delay or mental retardation. Am J Med Genet A 140(19):2063-2074

13. Ropers HH (2008) Genetics of intellectual disability. Curr Opin Genet Dev 18(3):241-250

14. Tarpey PS, Raymond FL, O'Meara S et al (2007) Mutations in CUL4B, which encodes a ubiquitin E3 ligase subunit, cause an $X$-linked mental retardation syndrome associated with aggressive outbursts, seizures, relative macrocephaly, central obesity, hypogonadism, pes cavus and tremor. Am J Hum Genet 80(2):345-352
15. Tarpey PS, Smith R, Pleasance E et al (2009) A systematic, large scale resequencing screen of the $X$ chromosome coding exons in mental retardation. Nat Genet 41(1):535-543

16. Turner G, Partington M (2000) Recurrence risks in undiagnosed mental retardation. J Med Genet 37(12):E45

17. Villard $L$ (2007) MECP2 mutations in males. J Med Genet 44(7):417-423 